\title{
Pacific
}

Journal of

Mathematics

\section{SINGULAR MODULI SPACES OF STABLE VECTOR BUNDLES} ON $\mathbf{P}^{3}$

\author{
ROSA M. MIRÓ-ROIG
}

Volume 172 No. 2 


\title{
SINGULAR MODULI SPACES OF STABLE VECTOR BUNDLES ON $\mathbf{P}^{3}$
}

\author{
Rosa M. Miró-RoIG
}

The goal of this paper is to give an example of singular moduli space of rank 3 stable vector bundles on $P^{3}$.

\section{Introduction.}

In $1977 / 78$, M. Maruyama proved the existence of a moduli scheme $M_{\mathbf{P}^{n}}\left(r ; c_{1}, \ldots, c_{\min (r, n)}\right)$ parametrizing isomorphic classes of rank $\mathrm{r}$ stable vector bundles on $\mathbf{P}^{n}$ with given Chern classes $c_{1}, \ldots, c_{\min (n, r)}$ (cf. [M1, M2]). The goal of this note is to give, to the best of my knowledge, the first example of singular moduli space of stable vector bundles on $\mathbf{P}^{3}$. It has been motivated by a recent work of Ancona and Ottaviani where they show that the moduli space $M I_{\mathbf{P}^{5}}(k)$ of stable instanton bundles on $\mathbf{P}^{5}$ with quantum number $\mathrm{k}=3$ or 4 is singular. Moreover they claim that $M I_{\mathbf{P}^{5}}(3)$ and $M I_{\mathbf{P}^{5}}(4)$ are the first examples of singular moduli spaces of stable vector bundles on projective spaces (cf. [AO]). Ancona-Ottaviani's result together with the well known fact that $M_{\mathbf{P}^{2}}\left(r ; c_{1}, c_{2}\right)$ is a smooth quasi-projective variety of dimension $2 r c_{2}-(r-1) c_{1}^{2}+1-r^{2}$ gives rise the following question:

Is there any example of singular moduli space of stable vector bundles on $\mathbf{P}^{3}$ ?

As I pointed out before my aim is to give an affirmative answer to this question (cf. Theorem 2.10).

\section{Preliminaries.}

In this section we recall some well known results needed later on.

1.1. Let $\mathrm{H}(18,39)$ be the open subscheme of $\mathrm{Hilb}_{k}^{3}$ parametrizing smooth connected curves $C \subset \mathbf{P}^{3}$ of degree 18 and genus 39. (See [EF] for a precise description of $\mathrm{H}(18,39)$.) Let $H_{1} \subset H(18,39)$ be the 72-dimensional irreducible, generically smooth component whose general point parametrizes an arithmetically Cohen-Macaulay curve $X \subset \mathbf{P}^{3}$ having a locally free resolution of the following type:

$$
0 \rightarrow \mathcal{O}(-7)^{4} \rightarrow \mathcal{O}(-6)^{4} \oplus \mathcal{O}(-4) \rightarrow I_{X} \rightarrow 0 .
$$


Let $H_{2} \subset H(18,39)$ be the 72-dimensional irreducible, generically smooth component whose general point parametrizes an arithmetically Cohen-Macaulay curve $Y \subset \mathbf{P}^{3}$ having a locally free resolution of the following type:

$$
0 \rightarrow \mathcal{O}(-6)^{2} \oplus \mathcal{O}(-8) \rightarrow \mathcal{O}(-5)^{4} \rightarrow I_{Y} \rightarrow 0 .
$$

It is well known that there exits an irreducible subset $H=H_{1} \cap H_{2} \subset$ $H(18,39)$ of dimension 71 whose general point parametrizes an arithmetically Buchsbaum curve $C \subset \mathbf{P}^{3}$ having a locally free resolution of the following type:

$$
0 \rightarrow \mathcal{O}(-8) \rightarrow \mathcal{O}(-7)^{4} \oplus \mathcal{O}(-8) \rightarrow \mathcal{O}(-6)^{4} \oplus \mathcal{O}(-4) \rightarrow I_{C} \rightarrow 0 .
$$

1.2. Remark. For all curve $Z \in H_{1} \cup H_{2}, \omega_{Z}(2)$ is globally generated. From now on, for all curve $Z \in H_{1} \cup H_{2}$, we set $\alpha:=\operatorname{dim} H^{0}\left(\omega_{Z}(2)\right)(=74$; by Riemann-Roch's Theorem).

1.3. Fact. Let $0 \rightarrow E \rightarrow F \rightarrow G \rightarrow 0$ be an exact sequence of vector bundles. Then, we have the following exact sequence involving alternating and symmetric powers:

$$
0 \rightarrow S^{q} E \rightarrow S^{q-1} E \otimes F \rightarrow \ldots . \rightarrow E \otimes \Lambda^{q-1} F \rightarrow \Lambda^{q} F \rightarrow \Lambda^{q} G \rightarrow 0 .
$$

1.4. Hoppe's criterion for the stability of a vector bundle. Let $X$ be a projective manifold with $\operatorname{Pic}(X) \cong \mathbf{Z}$ and let $E$ be a vector bundle on $X$. If $H^{0}\left(X,\left(\Lambda^{q} E\right)_{\text {norm }}\right)=0$ for $1 \leq q \leq r k(E)-1$, then $E$ is stable. As usual, given a vector bundle $E$ on $X$, we denote by $E_{\text {norm }}$ the twist of $E$ whose first Chern class $c_{1}$ verifies $-r k(E)+1 \leq c_{1} \leq 0$.

\section{Main results.}

2.1. Let us call $\mathcal{L}_{1}$ the irreducible family of sheaves $E$ on $\mathbf{P}^{3}$ constructed as an extension:

$$
\sigma=\left(\sigma_{1}, \ldots, \sigma_{\alpha}\right): \quad 0 \rightarrow \mathcal{O}^{\alpha} \rightarrow E(1) \rightarrow I_{X}(2) \rightarrow 0
$$

where $X \in H_{1}$ and $\sigma_{1}, \ldots, \sigma_{\alpha} \in H^{0}\left(\omega_{X}(2)\right) \cong \operatorname{Ext}^{1}\left(I_{X}(2), \mathcal{O}\right)$ are general global sections which generate the sheaf $\omega_{Z}(2)$ everywhere.

It is easy to see that $E$ is a vector bundle on $\mathbf{P}^{3}$ of rank $\alpha+1$.

2.2. Let us call $\mathcal{L}_{2}$ the irreducible family of sheaves $F$ on $\mathbf{P}^{3}$ constructed as an extension:

$$
\lambda=\left(\lambda_{1}, \ldots, \lambda_{\alpha}\right): \quad 0 \rightarrow \mathcal{O}^{\alpha} \rightarrow F(1) \rightarrow I_{Y}(2) \rightarrow 0
$$

where $Y \in H_{2}$ and $\lambda_{1}, \ldots, \lambda_{\alpha} \in H^{0}\left(\omega_{Y}(2)\right) \cong \operatorname{Ext}^{1}\left(I_{Y}(2), \mathcal{O}\right)$ are general global sections which generate the sheaf $\omega_{Z}(2)$ everywhere. 
Again it is easy to see that $F$ is a vector bundle on $\mathbf{P}^{3}$ of rank $\alpha+1$. 2.3. And let $\mathcal{L} \subset \mathcal{L}_{1} \cap \mathcal{L}_{2}$ be the irreducible family of sheaves $G$ on $\mathbf{P}^{3}$ constructed as an extension:

$$
\mu=\left(\mu_{1}, \ldots, \mu_{\alpha}\right): \quad 0 \rightarrow \mathcal{O}^{\alpha} \rightarrow G(1) \rightarrow I_{C}(2) \rightarrow 0
$$

where $C \in H \subset H_{1} \cap H_{2}$ and $\mu_{1}, \ldots, \mu_{\alpha} \in H^{0}\left(\omega_{C}(2)\right) \cong \operatorname{Ext}^{1}\left(I_{C}(2), \mathcal{O}\right)$ are general global sections which generate the sheaf $\omega_{Z}(2)$ everywhere.

Again it is easy to see that $G$ is a vector bundle on $\mathbf{P}^{3}$ of rank $\alpha+1$.

\section{Proposition 2.4.}

(1) A general vector bundle $E \in \mathcal{L}_{1}$ has a locally free resolution of the following type:

$$
0 \rightarrow \mathcal{O}(-5)^{4} \rightarrow \mathcal{O}(-4)^{4} \oplus \mathcal{O}(-2) \oplus \mathcal{O}^{\alpha} \rightarrow E(1) \rightarrow 0 .
$$

(2) A general vector bundle $F \in \mathcal{L}_{2}$ has a locally free resolution of the following type:

$$
0 \rightarrow \mathcal{O}(-6) \oplus \mathcal{O}(-4)^{2} \rightarrow \mathcal{O}(-3)^{4} \oplus \mathcal{O}^{\alpha} \rightarrow F(1) \rightarrow 0 .
$$

(3) A general vector bundle $G \in \mathcal{L}$ has a locally free resolution of the following type:

$$
0 \rightarrow \mathcal{O}(-6) \rightarrow \mathcal{O}(-6) \oplus \mathcal{O}(-5)^{4} \rightarrow \mathcal{O}(-4)^{4} \oplus \mathcal{O}(-2) \oplus \mathcal{O}^{\alpha} \rightarrow G(1) \rightarrow 0 .
$$

Proof. (1) From the exact sequence:

$$
0 \rightarrow \mathcal{O}^{\alpha} \rightarrow E(1) \rightarrow I_{X}(2) \rightarrow 0
$$

and the locally free resolution of $I_{X}(2)$ (See 1.1):

$$
0 \rightarrow \mathcal{O}(-5)^{4} \rightarrow \mathcal{O}(-4)^{4} \oplus \mathcal{O}(-2) \rightarrow I_{X}(2) \rightarrow 0
$$

we get the following commutative diagram:
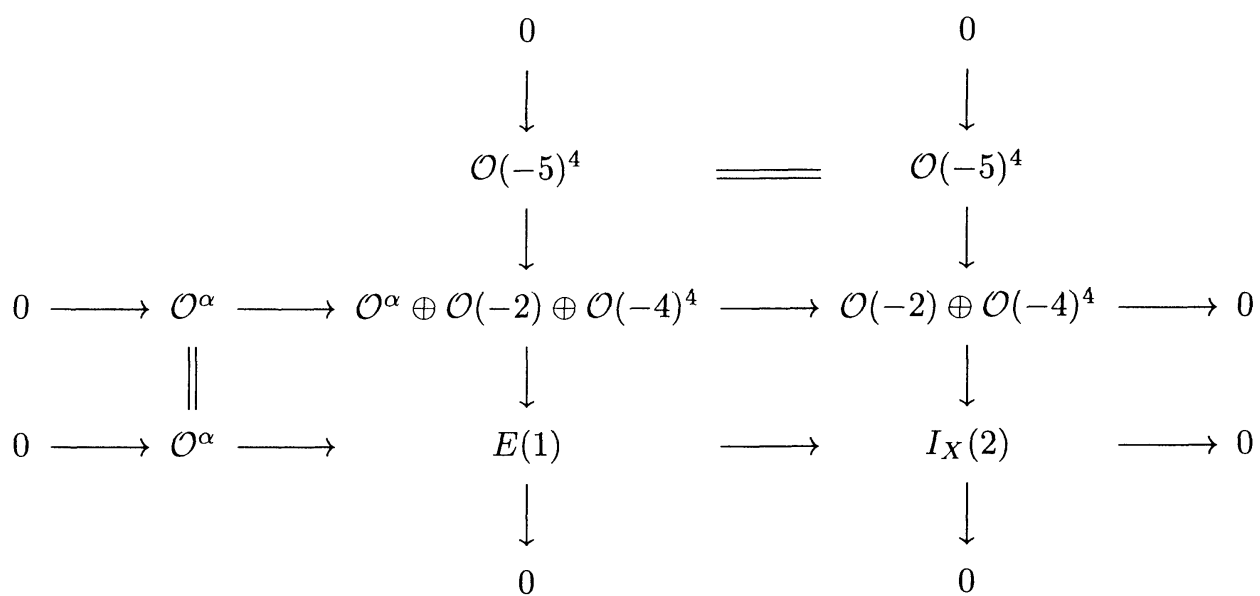
which gives what we want.

(2) and (3) Analogous.

Corollary 2.5. Given a vector bundle $E \in \mathcal{L}_{1} \cup \mathcal{L}_{2}, E(t)$ is globally generated for all $t \geq 5$.

2.6. Let $\mathcal{F}_{1}$ be the irreducible family of rank 3 vector bundles $P$ on $\mathbf{P}^{3}$ defined as the cokernel:

$$
0 \rightarrow \mathcal{O}(-5)^{\alpha-2} \underset{s_{1}, \ldots, s_{2}}{\longrightarrow} E \rightarrow P \rightarrow 0
$$

where $E \in \mathcal{L}_{1}$ and $s_{i} \in H^{0}(E(5))$ are general global sections of $E(5)$.

2.7. Let $\mathcal{F}_{2}$ be the irreducible family of rank 3 vector bundles $Q$ on $\mathbf{P}^{3}$ defined as the cokernel:

$$
0 \rightarrow \mathcal{O}(-5)^{\alpha-2} \underset{f_{1}, \ldots, f_{2}}{\longrightarrow} F \rightarrow Q \rightarrow 0
$$

where $F \in \mathcal{L}_{2}$ and $f_{i} \in H^{0}(F(5))$ are general global sections of $F(5)$.

2.8. Let $\mathcal{F} \subset \mathcal{L}_{1} \cap \mathcal{L}_{2}$ be the irreducible family of rank 3 vector bundles $R$ on $\mathbf{P}^{3}$ defined as the cokernel:

$$
0 \rightarrow \mathcal{O}(-5)^{\alpha-2} \underset{g_{1}, \ldots, g_{2}}{\longrightarrow} G \rightarrow R \rightarrow 0
$$

where $G \in \mathcal{L}$ and $g_{i} \in H^{0}(G(5))$ are general global sections of $G(5)$.

\section{Proposition 2.9.}

(1) A general vector bundle $P$ of $\mathcal{F}_{1}$ is a rank 3 stable vector bundle on $\mathbf{P}^{3}$ with Chern classes $(287,42065,4195775)$.

(2) A general vector bundle $Q$ of $\mathcal{F}_{2}$ is a rank 3 stable vector bundle on $\mathbf{P}^{3}$ with Chern classes $(287,42065,4195775)$.

(3) A general vector bundle $R$ of $\mathcal{F}$ is a rank 3 stable vector bundle on $\mathbf{P}^{3}$ with Chern classes $(287,42065,4195775)$.

Proof. (1) Using the exact sequence:

$$
0 \rightarrow \mathcal{O}(-5)^{\alpha-2} \rightarrow E \rightarrow P \rightarrow 0
$$

and the locally free resolution of $E$ given in Proposition 2.4(1) we get:

$$
c_{t}(P)=(1-3 t)(1-t)^{74} /\left((1-6 t)^{4}(1-5 t)^{68}\right) .
$$

Hence $c_{1}(P)=287, c_{2}(P)=42065$ and $c_{3}(P)=4195775$. 
Let us see that $P$ is stable. Using Hoppe's criterion we need to prove that $H^{0}(P)_{\text {norm }}=H^{0}\left(\Lambda^{2} P\right)_{\text {norm }}=0$. Since $c_{1}(P)>0$ and $c_{1}\left(\Lambda^{2} P\right)>0$, we have $(P)_{\text {norm }}=P(\lambda)$ and $\left(\Lambda^{2} P\right)_{\text {norm }}=\left(\Lambda^{2} P\right)(\rho)$ for some $\rho, \lambda \leq-1$. So it siffices to prove that $H^{0}(P)(-1)=H^{0}\left(\Lambda^{2} P\right)(-1)=0$.

Using the exact sequence $\left({ }^{*}\right)$ and the locally free resolution of $E$ given in Proposition 2.4(1) we easily get that $H^{0} E(-1)=H^{0} P(-1)=0$. Again using the exact sequence $\left(^{*}\right)$ and taking wedge powers we get the exact sequence

$$
0 \rightarrow S^{2} \mathcal{O}(-5)^{\alpha-2} \rightarrow \mathcal{O}(-5)^{\alpha-2} \otimes E \rightarrow \Lambda^{2} E \rightarrow \Lambda^{2} P \rightarrow 0
$$

cutting in short exact sequences we get $H^{0}\left(\Lambda^{2} P\right)(-1)=H^{0}\left(\Lambda^{2} E\right)(-1)=0$ where the last equality follows from the locally free resolution of $E$ given in Proposition 2.4(1) taking wedge powers and cutting in short exact sequences.

(2) and (3) are analogous.

Theorem 2.10. The moduli space $M_{\mathbf{P}^{3}}(3 ;-1,14609,1917791)$ is singular.

Proof. We have constructed two irreducible families $\mathcal{F}_{1}$ and $\mathcal{F}_{2}$ of rank 3 stable vector bundles on $\mathbf{P}^{3}$ with Chern classes $(287,42065,4195775)$ which meets along an irreducible family $\mathcal{F}$. Hence in order to see that $M:=$ $M_{\mathbf{P}^{3}}(-1,14609,1917791) \cong M_{\mathbf{P}^{3}}(-287,42065,4195775)$ is singular it is enough to prove that $\mathcal{F}_{1}$ and $\mathcal{F}_{2}$ belongs to two different components of $M$. Using proposition 2.9 and 2.4 we get:

(1) If $P$ is a general vector bundle of $\mathcal{F}_{1}$ then:

$$
\begin{aligned}
& H_{*}^{1} P=H^{3} P(3)=0 \\
& h^{0} P(3)=1+10 \alpha, h^{2} P(3)=0 .
\end{aligned}
$$

(2) If $Q$ is a general vector bundle of $\mathcal{F}_{2}$ then:

$$
\begin{aligned}
& H_{*}^{1} Q=H^{3} Q(3)=0 \\
& h^{0} Q(3)=10 \alpha, h^{2} Q(3)=1 .
\end{aligned}
$$

Therefore, by semicontinuity $\mathcal{F}_{1}$ and $\mathcal{F}_{2}$ are contained in different components of $M$ which gives what we want.

\section{References}

[AO] V. Ancona and G. Ottaviani, On moduli of instanton bundles on $\mathbf{P}^{2 n+1}$, Pacific J. Math., to appear.

[EF] Ph. Ellia and M. Fiorentini, Défaut de postulation et singularités du schéme de Hilbert, Ann. Univ. Ferrara, 30 (1984), 185-198. 
[H] H. Hoppe, Generischer spaltungstypum un zweite Chernklasse stabiler Vektorraumbündel vom rang 4 auf $\mathbf{P}^{4}$, Math. Z., 187 (1984), 345-360.

[M1] M. Maruyama, Moduli of stable sheaves, I, J. Math. Kyoto Univ., 17 (1977), 91-126.

[M2] _ Moduli of stable sheaves, II, J. Math. Kyoto Univ., 18 (1978), 557-614.

Received September 13, 1993. The author was partially supported by DGICYT PB940850.

Department Algebra y geometría

Universidad de Barceloan, 08007 Barcelona, Spain

E-mail address: miro@cerber.ub.es 
Rosa M. Miró-Roig, Singular moduli spaces of stable vector bundles on $\mathbf{P}^{3}$

Hitoshi Moriyoshi and Toshikazu Natsume, The Godbillon-Vey Cyclic Cocycle and Longitudinal Dirac Operators

J.C. Naranjo, The positive dimensional fibres of the Prym map

Artur Nicolau and Arne Stray, Nevanlinna's coefficients and Douglas al-

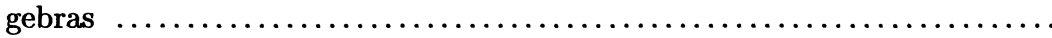

K.K. Park, Entropy of a skew product with a $Z^{2}$-action $\ldots \ldots \ldots \ldots \ldots .227$

María Cristina Pereyra, Sobolev spaces on Lipschitz curves ......... 553

T. Sano, Commuting co-commuting squares and finite dimensional Kac algebras

H.B. Thompson, Second order ordinary differential equations with fully nonlinear two point boundary conditions

H.B. Thompson, Second order ordinary differential equations with fully nonlinear two point boundary conditions II

F. $\mathrm{Xu}$, The flat part of non-flat orbifolds

Hidenobu Yoshida, A type of uniqueness for the Dirichlet problem on a half-space with continuous data 


\section{PACIFIC JOURNAL OF MATHEMATICS}

Volume $172 \quad$ No. $2 \quad$ February 1996

On the failure cycles for the quadratic normality of a projective variety

307

EdoARdo BaLlico

On the minimal free resolution of general embeddings of curves

315

EdOARDO BALlico

On normality of the closure of a generic torus orbit in $G / P$

321

ROMUALD DABROWSKI

Paragroupe d'Adrian Ocneanu et algèbre de Kac

331

MARIE-Claude DAVID

Irreducibility and dimension theorems for families of height 3 Gorenstein algebras

SuSAN J. DiESEL

On the cohomology of the Lie algebra $L_{2}$

ALICE FIALOWSKI

Generic differentiability of convex functions on the dual of a Banach space

John R. Giles, P. S. Kenderov, WarRen Brian Moors and S. D.

SCIFFER

Moon hypersurfaces and some related existence results of capillary hypersurfaces

without gravity and of rotational symmetry

FEI-TSEN LIANG

Stable relations. II. Corona semiprojectivity and dimension-drop $C^{*}$-algebras

TERRY ATHERTON LORING

Singular moduli spaces of stable vector bundles on $\mathbf{P}^{3}$

Rosa M. MIRó-ROIG

The Godbillon-Vey cyclic cocycle and longitudinal Dirac operators

Hitoshi MoRIYOSHI and TOSHIKAZU NATSUME

Nevanlinna's coefficients and Douglas algebras

ARTUR NiCOLAU and ARNE STRAY

Sobolev spaces on Lipschitz curves

MARÍA CRISTINA PEREYRA

A type of uniqueness for the Dirichlet problem on a half-space with continuous data 